\title{
Characterisation of Large Changes in Wind Power for the Day-Ahead Market Using a Fuzzy Logic Approach
}

\author{
Giovanna Martínez-Arellano • Lars Nolle • Richard Cant • Ahmad \\ Lotfi · Christopher Windmill
}

Received: date / Accepted: date

\begin{abstract}
Wind power has become one of the renewable resources with a major growth in the electricity market. However, due to its inherent variability, forecasting techniques are necessary for the optimum scheduling of the electric grid, specially during ramp events. These large changes in wind power may not be captured by wind power point forecasts even with very high resolution Numerical Weather Prediction (NWP) models. In this paper, a fuzzy approach for wind power ramp characterisation is presented. The main benefit of this technique is that it avoids the binary definition of ramp event, allowing to identify changes in power output that can potentially turn into ramp events when the total percentage of change to be considered a ramp event is not met. To study the application of this technique, wind power forecasts were obtained and their corresponding error estimated using Genetic Programming (GP) and Quantile Regression Forests. The error distributions were incorporated into the characterisation process, which according to the results, improve significantly the ramp capture. Results are presented
\end{abstract}

G. Martínez-Arellano, R. Cant, A. Lotfi, C.Windmill School of Science and Technology

Nottingham Trent University

Clifton Lane, NG11 8NS, UK

E-mail: N0204624@ntu.ac.uk

L. Nolle

Department of Engineering Sciences

Jade University of Applied Science

Friedrich-Paffrath-Str. 101, 26389 Wilhelmshaven, Germany

E-mail: lars.nolle@jade-hs.de using colour maps, which provide a useful way to interpret the characteristics of the ramp events.

Keywords Wind power forecasting - ramp events · genetic programming · uncertainty

\section{Introduction}

During the last decades, there has been an increasing interest on the use of renewable energy sources to decrease $\mathrm{CO}_{2}$ emissions. As a result, efforts have been made to integrate renewable energy into the electric grid. Wind power has had the strongest growth in the electricity markets over the last years [1]. However, wind as a source of energy is specially challenging due to its variability and intermittence. Integrating large amounts of wind power into the electric grid requires the implementation of forecasting tools able to provide information to the grid operator for the day-ahead market and in real-time.

In most markets, one day before the real time operation, grid operators set a schedule defining which units will supply the expected demand for the next day at each hour. Backup units are also allocated to deal with unexpected shutdowns of the scheduled units. These decisions are part of what is called the Unit Commitment (UC) problem, and depending on the market, these can be taken early in the morning or by mid-day on the day before. In order to include wind power on the day-ahead market, a forecast on the short term (up to 48 hours into 
the future) of the hourly wind power production would need to be provided to the grid operator.

Wind power forecasting models are nowadays used by some grid operators for UC, however, existing tools need to be improved to be able to handle extreme situations related to wind power generation [2]. These extreme situations, also known as ramp events, may be related to specific meteorological events, such as cold fronts or high pressure levels, which can produce drastic and unexpected increases or drops in the level of power production of a wind farm [3]. These sudden increases or drops may happen within a couple of minutes or a couple of hours. An early detection of the possibility of these events happening would let the grid operator prepare the most appropriate backup units according to the characteristics of the event. Different types of backup units respond at different speeds so an estimation of the intensity and the time scale of an event is important. The prediction of ramp events is commonly addressed using either point forecasts, obtained by running high resolution numerical models, or ensemble forecasts, which are obtained by running NWP models with different perturbations of the initial state [25]. Despite high resolution numerical runs being avoided in ensemble forecasting, ensembles are still computationally expensive. NWP models may have misplacement errors and therefore the closest point of the grid might not always be the best reference point from the numerical model. In addition, current ramp characterisation techniques are based on a crisp binary definition, which can leave out events that did not quite meet the amount of change expected but that could be equally important to consider.

This paper puts together a wind power forecasting approach and a ramp characterisation technique in order to address the weaknesses introduced previously and extending the preliminary results obtained in [28]. Wind power forecasts are first obtained by using GP as a final downscaling procedure at different points of the mesoscale grid, producing a set of models and possible wind power realisations for a wind farm located in a semi-complex terrain in Galicia, Spain. The error of each forecast is quantified and incorporated into the characterisation process, which consists of a set of fuzzy rules. These rules are based on the assumption that changes in power output that do not meet the strict definition of ramp event can be equally important and damaging and should not be ignored. To differentiate between highly probable events and events with lower probability, a score is given that is the result of the application of fuzzy rules defined based on post experience. The rest of the paper is organised as follows: Section 2 presents previous work related to wind power and ramp event prediction. Section 3 provides detail about the proposed approach for wind power forecasting and uncertainty estimation using Quantile Regression Forests. Section 4 discusses the ramp characterisation approach and the results. Finally, Section 5 draws the paper to a close giving conclusions and future work.

\section{Related Work}

In order to characterise large changes in power output, one must first obtain a wind power forecast. Most shortterm forecasting tools are based on Numerical Weather Prediction (NWP) models, as these can capture the atmospheric flow more accurately than methods based purely on historical observations [4]. These models can be classified into three types. Firstly global models, e.g. the Global Forecast System (GFS) from the National Oceanic and Atmospheric Administration in the USA (NOAA) [5], which produce low space resolution forecasts of the entire globe. Secondly, mesoscale models, which produce forecasts with a space resolution of up to one square $\mathrm{km}$ in a specific region, and finally local models, with the highest space resolution. Models such as the High Resolution Limited Area Model (HIRLAM) have been used for short term forecasting $[6,7]$. In [8], the Weather Research and Forecasting (WRF) mesoscale model [9] is used for quantifying wind uncertainty for the day ahead and for studying the economic impact of large amounts of wind power in the electric grid. In [10] the use of global physical models together with Neural networks and autoregressive models was proposed for short term forecasting. Neural networks were found to be the approach that provided better results. In [11] the ETA model has been applied to wind speed forecasting in Sweden.

Two main approaches are derived from NWP predictions; the physical approach, which translate wind forecasts at a certain grid point using mathematical descriptions of the physical processes relevant to the translation, and the statistical approach, which combine inputs such as wind speed, wind direction, temperature together with online measurements to either estimate wind speed or wind generation [12]. Further 
details of these methods can be found in reviews of wind power prediction $[4,12-14]$. In any case, the resulting forecast can either be a point forecast, which provides an exact amount of the hourly power output, or an ensemble or scenario forecast, which provides a set of possible wind power estimates.

The point forecast approach generates a power prediction based on the result of one run of the numerical model to predict wind speed at a close point from the location of interest (usually a location at the farm). A statistical downscaling technique may be applied to correct the local physical attributes that could not be modeled. These methods included a Kalman filter approach [15], ANN approach [19], bias correction methods and a combination of these. In [16], a WRF model is implemented together with the Kalman filter method for wind speed and wind power forecasting for a wind farm in China. Kalman filter approaches have also been applied in [17] and [18], as post-processing tools for correcting the bias of WRF wind speed predictions, reducing significantly the size of the training set, compared to ANN based methods. The majority of these approaches require the numerical weather prediction model to run at a very high resolution, which is time and resource consuming. Salcedo-Sanz et al. use a neural network approach (ANN) to perform the final downscaling from the MM5 mesoscale model to the observation sites avoiding the execution of the numerical model at high resolutions [19]. However, neural networks behave as black boxes, which do not provide internal information about the model that was found. They also need a significant amount of training data to ensure generalisation. The same forecasting model used in [19] was implemented in [20] replacing the ANN with a support vector machine (SVM) approach. Although point forecasts does not provide a quantification of the wind power uncertainty directly, different methods can be applied to estimate it. These methods are local quantile regression, local Gaussian modeling, the Nadaraya-Watson estimator, kernel density estimation for $p d f$ computation, among others [41].

The ensemble forecast approach generates a set of forecasts by running the NWP model varying the initial conditions $[21,22]$ or the physical parameterisations [23]. Due to the computational cost of ensembles, these are run at a lower resolution to reduce the cost. Greaves et al. present a study where NWP models are used for wind power ramp forecasting [24]. NWP forecasts from two sources are used to improve the characterisation of the timing uncertainty of the ramps. Bossavy et al. proposed the use of NWP ensembles for wind power ramp predictions [25]. The ensembles are used to improve the timing error of ramp forecasts. According to Cutler et al, the timing or phase error of numerical models can be addressed taking into account a wider area of the NWP grid, not only the closest point to the observation site, due to the misplacement errors that the numerical model may have [26]. Using a set of grid points could generate an ensemble prediction which is taking into consideration the horizontal error of the numerical model. In [28], a ramp detection algorithm based on wind power models generated by genetic programming (GP) is presented. Preliminary results show a potential use of neighbour points as a way to quantify the uncertainty of ramp events.

The majority of ramp event studies define a ramp as a specific percentage of change in power output within a specific time window (binary classification). The disadvantage of this definition is that slightly lower changes might not be identified that may be equally important to the grid operator. Gallego et al. present a ramp forecasting approach using wavelets [27]. This approach avoids using a fix change percentage, analysing the power forecast at different magnitudes of change and different time windows. Despite their promising results, the application on the day-ahead market is not addressed in depth nor how this could allow end users to interpret the different ramp intensities. This paper explores a new approach for characterising ramp events which minimises the sentisitivity of the binary classification and provides more insight about the characteristics of the event, providing a visual representation that could benefit system operators in the understanding of the severity of events to come.

\section{Proposed method for wind power forecasting}

In order to predict the wind power output of a wind farm at each hour for the next day and identify the possible future ramp events, two major steps are carried out in this approach. First, wind speeds at a close point to the wind farm are obtained using the Weather Research and Forecasting - Advanced Research WRF (WRF-ARW) numerical weather prediction model. Secondly, wind speed predictions and wind power observations at the farm are used by a GP based algorithm 
to obtain a model which best represents the relationship between the wind speed predicted by the numerical model and the actual power output produced by the wind farm.The model found can be applied to newly available wind speed forecasts to predict the power output of the wind farm.

\subsection{Wind speed prediction}

WRF-ARW is a non-hydrostatic limited area model from the National Center of Atmospheric Research in the USA (NCAR) [32]. The model solves a system of differential equations that represent the dynamics of the atmospheric flow, except that it does not take into account the ocean-land interactions. The solver uses a second- or third -order Runge Kutta time integration scheme with a small time step for the acoustic and gravity-wave modes. Its prognostic variables include velocity components $u$ and $v$ in Cartesian coordinate, vertical velocity $w$, potential temperature perturbations, perturbation geopotential, perturbation surface pressure of dry air, turbulent kinetic energy, and scalars (water vapor mixing ratio, rain/snow mixing ratio, cloud water/ice mixing ratio, etc.). This mesoscale model has multiple physics options classified in different categories, which are (1) microphysics, (2) cumulus parameterisation, (3) land surface model, (4) planetary boundary layer and (5) radiation. To reduce as much as possible the execution time of the model, a CUDA based version of the WSM5 (WRF Single Moment 5 Cloud) microphysics kernel was used [33]. The rest of the physics options used are shown in Table 1.

Table 1: Physics options used in the WRF model

\begin{tabular}{ll}
\hline Domains & $\begin{array}{l}\text { 2 nested domains } \\
\text { nonhydrostatic Euler equations } \\
\text { Longwave Radiation }\end{array}$ \\
$\begin{array}{l}\text { rapid radiative transfer model } \\
\text { (RRTM) }\end{array}$ \\
$\begin{array}{l}\text { Shortwave Radiation } \\
\text { Surface Layer }\end{array}$ & MM5 similarity \\
Boundary Layer & YSU scheme \\
Cumulus & Kain-Fritsch (new Eta) scheme \\
\hline
\end{tabular}

In this research, the initial state was generated using GFS forecasts and terrestrial data. GFS runs four times a day, at 00Z, 06Z, 12Z and 18Z (Zulu time). At each run, it produces low resolution forecasts. This means the entire globe is divided into a grid of usually $1^{\circ} \times 1^{\circ}$ producing forecasts at each of the intersection points of the grid. Each run predicts up to 16 days in advance with a three-hour time step. For this study, the runs from $06 \mathrm{Z}$ were used, taking into account that forecasts need to be provided to the operator before noon for the day ahead. From each run, only the first 48 hours of the complete forecasted horizon were considered. For each GFS run, a WRF-ARW run was executed, producing a higher resolution forecast in time and space. Those values forecasted for the next day (19 to 42 hours into the future), as shown in Figure 1, are the values of interest in this study.

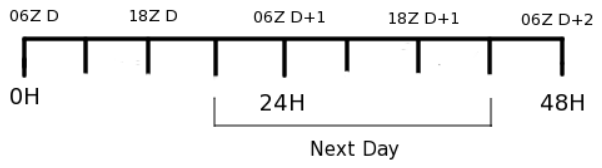

Fig. 1: WRF-ARW 48 hour horizon in a 06Z run started at day $\mathrm{D}$. The next day forecast horizon corresponds to those values 19 to 42 hours into the future

WRF-ARW, as other mesoscale models, allows nesting. This means the model can run at different resolutions or domains; one contained into the other, where the inner domains have a higher resolution in a smaller area. Figure 2 shows the domain settings that were used in this work. As shown in the figure, the model was set to run in two domains. The first domain, which covers a major part of Spain, has a resolution of $30 \mathrm{~km} \mathrm{x}$ $30 \mathrm{~km}$ and results from the first integration of the WRF model from the GFS grid $(111 \mathrm{~km} \times 78 \mathrm{~km})$. The second domain, which is centered on Galicia, the area of interest, has a resolution of $10 \mathrm{~km} \times 10 \mathrm{~km}$ and is obtained by a second model integration that uses the first domain as boundary conditions. A third domain was considered, but the computational cost of running at $3 \mathrm{~km}$ resolution was considered too high. From the model output, wind speed and wind direction forecasts from the closest point of the grid to the location of the wind farm are used for power prediction. 


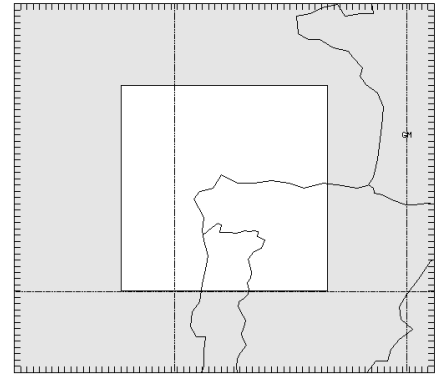

Fig. 2: WRF-ARW two domain setting. The second domain (white area) is centered in the point of interest, which is Galicia, Spain.

\subsection{Wind Power Prediction using Genetic Programming}

In a previous work [34], GP was used as a final downscaling step from the mesoscale model to the location of the observation site for wind speed forecasting. Results obtained showed the ability of the GP approach to model the relationship between the forecasts of the WRF-ARW model and the observations at specific sites. Wind speed forecasts can be easily converted into wind power forecasts using the power curve provided by the wind turbine manufacturer. However, the performance of the wind turbines depends on the characteristics of the place where they are located. For this reason, the use of local wind power observations to estimate the power output of each turbine is suggested [35]. This paper revisits and extends the GP downscaling approach from [34] as a wind power prediction technique. While the input to the algorithm stays the same (numerical model predictions), the output changes to the total wind power produced by the wind farm.

Genetic Programming [29] is a biologically inspired computation technique based on the evolution of individuals over time, through events such as inheritance and mutation, which progressively refines them into better individuals. In GP, a population of programs (in a binary tree layout) is evolved, each program representing a set of instructions to solve a specific problem. GP, like nature, is a stochastic process, which cannot guarantee to find the global optimum but it is that randomness which can lead it to escape local optima, which deterministic methods may be captured by [30].

Symbolic regression via GP is a non-parametric, non-linear regression technique that looks for an ap-

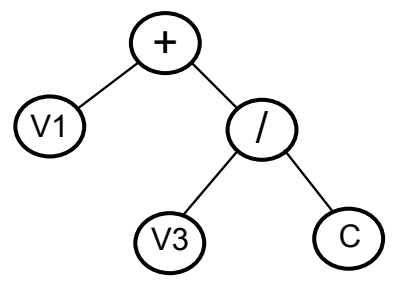

Fig. 3: Example of a tree expression of the program $v_{0}=v_{1}+v_{3} / C$.

propriate model structure and model parameters as opposed to classic regression, which assumes a certain model structure and estimates the optimal parameters that best fit a given sample of data [31].

As shown by the example in Figure 3, a GP tree is formed by a set of terminals and functions. The functions may be basic arithmetic operators $\{+,-, *, /\}$, standard mathematical functions (sine, cosine, logarithmic, exponential), logical functions or domain-specific functions. The terminals may be constants or any problemrelated variables. In wind power prediction, variables such as wind speed, wind direction, temperature, among others, may be relevant for the problem. The evolution process will be able to identify those variables that are relevant for the model.

Two basic genetic operators are used in order to create new individuals at each generation. One is crossover, which provides the means to generate new individuals from an existing population. It combines the material from two selected trees to generate two new trees. The second operator is mutation, which replaces a randomly selected subtree from a complete tree with a new randomly generated subtree. Every new individual in a population is a candidate for mutation, however this depends on a certain probability. The mutation probability is a parameter that needs to be tuned during experimentation and it is usually set to very small values.

In order to ensure generalisation, a parsimony pressure technique [31] is used, which penalises the fitness of a program according to its complexity (number of nodes in the tree), reducing the probability for it to be selected in future generations for crossover. The mathematical representation of the penalisation function used is shown on Equation 1. The first term of the fitness equation is the sum of the errors between the obtained output (new forecast) and the desired output (observations) in the test set (test set size $=s$ ). The second 
term is the complexity factor, where $\mathrm{t}$ is the number of nodes of the GP tested and $\mathrm{k}$ is a trade-off weight that allows to control the level of pressure of the complexity factor. A small value of $\mathrm{k}(\mathrm{e} . \mathrm{g} . k=0.1)$ would be translated into low complexity pressure, and higher values of $\mathrm{k}(\mathrm{e} . \mathrm{g} . k=1.0)$ will add a strong pressure to the penalisation.

$f=\frac{1}{s} \sum_{i=0}^{s} e(i)+k\left(\frac{\left(t^{2} \log _{2}(t)\right.}{s}\right)^{\frac{1}{2}}$

The GP approach was used to model the relationship between wind speed forecasts from the numerical model WRF-ARW and the power production of the farm. A starting point was to a single point from the mesoscale grid, the closest one as presented on [28] and subsequently extending it to the use of four neighbour points. For this study, data from the experimental wind farm, Sotavento, located in Galicia, Spain, was used [36]. This wind farm is composed of 24 wind turbines of 9 different models (each model with a different power curve) and has a total nominal power of 17.56 MW. The wind farm provides open access to wind speed and wind power observations. The wind speed observations are obtained by an anemometer situated at the wind farm at $45 \mathrm{~m}$ height. The day-ahead electricity market requires hourly forecasts to plan the commitment and dispatch of the power units. For this reason, mean hourly wind speeds from the farm where used.

In order to perform the experiments using the closest point from the grid, the training, validation and test sets were generated using four months of day-ahead WRF-ARW forecasts, from January to April 2012 (not consecutive days, due to missing global model data). Observations from the same period of time were obtained from the Sotavento wind farm. Therefore, for every hourly wind speed and direction forecast (at the closest point of the mesoscale grid to the wind farm), a wind power observation was associated. The first three months were used as a training/validation set and the fourth month as a test set.

As it can be observed in Figure 4, changes in the wind direction affect the energy production of the farm. When the wind blows in East Northeast direction (56.25 to 78.75 degrees) or in West Southwest direction (236.25 to 258.75 degrees), the maximum wind power output is achieved for high wind speeds. On the other hand, when the wind blows in North direction (348.75 to 11.25 de- grees) the power output is lower even for the higher wind speeds. For this reason, it is important to take into account both wind speed and wind direction as inputs for the GP.

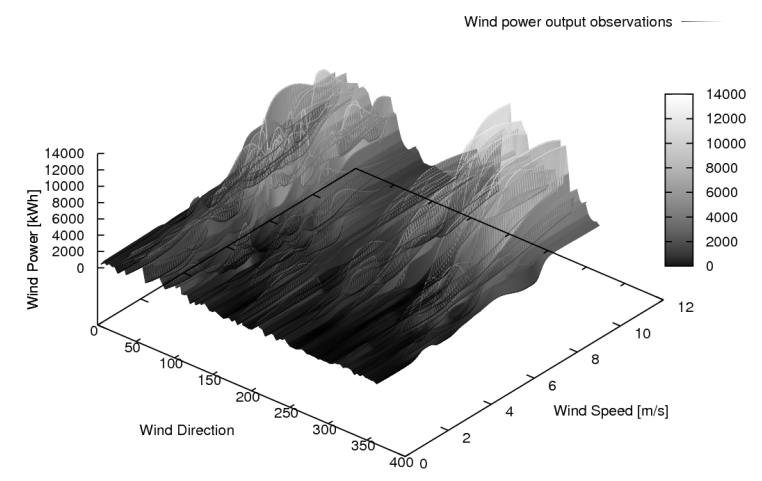

Fig. 4: Relationship between wind speed, wind direction and the power output at Sotavento wind farm for three months, January to March, 2012.

Once the data sets were ready, the parsimony pressure parameter was found experimentally and 50 runs of the algorithm were performed. The best model in 50 runs is applied to test data (Figure 5).

It can be observed in Figure 5 that the global trend is well captured, however, there is a time misplacement around hour 150, which is associated with a weakness of NWP modeling to forecast synoptic events like cold fronts and high or low pressure systems in the adequate position [26]. This misplacement could be addressed by taking into account a wider area from the mesoscale grid instead of taking only the closest point.

\subsection{Uncertainty Estimation of Wind Power Forecasts}

A point forecast like the one obtained can be used by the grid operator for the daily unit commitment. However, it has been shown that the point forecast is more useful if it is supplied with an uncertainty estimation [37]. This estimation can give the operator a better idea about the amount of backup needed. The total error of the wind power forecast can be the product of two sources. The first source of error is the error introduced by the numerical model. This error tends to 


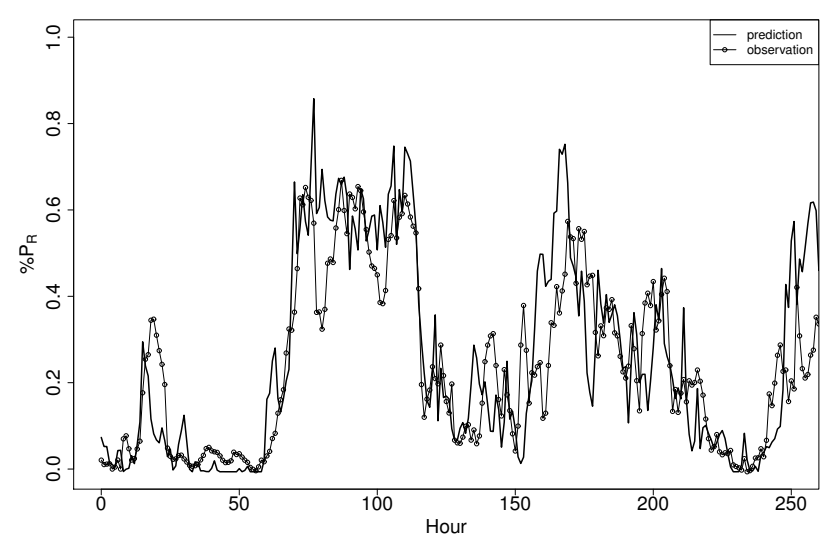

Fig. 5: Best Model found applied to test data (April 2012) and the real power output at Sotavento. Pressure parameter $k=2.0$.

increase as the forecasting horizon increases. The second source of error is the conversion process from wind speed to wind power. This process can depend on local conditions such as the roughness and orography of the wind farm location [38]. This type of error could be potentially decreased by the use of empirical power curves rather than the power curve provided by the manufacturer.

There are several approaches in the literature to quantify the uncertainty of wind power point forecast errors. In general, the error distribution is found by observing the behaviour of the error on past forecasts and by using explanatory variables which are additional information such as wind speed, wind direction, temperature, that can improve the understanding and thus the modeling of the error.

In order to anaylse the behaviour of the forecast error obtained with the GP model, histograms were used. Figure 6 shows the empirical distribution of the error in the training set for the first and last hour of the nextday horizon. Each hour of the horizon has been treated separately as a first attempt to study the use of the error estimation on the ramp characterisation. One can observe that the distribution of the error is different for the horizon $t+19$ and $t+42$. The shorter horizon shows a more distributed error while the larger horizon shows more overestimation errors, as the frequency of negative errors is higher. It can also be observed that the upper bound of the empirical distribution at $t+42$ is slightly higher. This means that at this horizon errors of $35 \%$ were observed, while at $t+19$ errors stayed lower than $30 \%$ of the nominal capacity of the farm.

Studies suggest that wind power forecast errors do not follow a normal distribution as wind speed forecast errors do. In fact, wind power error distributions have been found to have high kurtosis and skewness [37]. For this reason, an approach that makes no assumption of the distribution when estimating the uncertainty could be more appropriate. Quantile Regression Forests [39] is a non-parametric technique to estimate conditional quantiles for high dimensional predictor variables of a response variable. The detailed description of this method is out of the scope of this paper but can be found in [39]. By looking into the training errors of the GP model, error quantiles were obtained by applying the quantile regression forest method for each hour of the next-day horizon. The distribution of the error was found using wind power, wind speed and wind direction forecasts as explanatory variables. Figure 7 presents the point forecasts for the first four days of the April test set as well as the prediction intervals $(10 \%, 20 \%, \ldots$, $90 \%$ ) obtained using the error distributions found.

The distributions found were applied to the test set which corresponds to one month after the last training point. It is important to consider that the distributions found are considering a small set of history observations, which could probably indicate that these are only accurate for a short period after the last training point. In order to assess the influence of the sample size on the quality of the estimated intervals, reliability diagrams were used. These reliability diagrams provide information about the deviation of the actual coverage $\left(\hat{a}^{\alpha}\right)$ of the forecasted intervals from the nominal proportions $(\alpha)$. This deviation is defined as

$\alpha-\hat{a}^{(\alpha)}=\alpha-\frac{z^{(\alpha)}}{N}$

where $N$ is the total number of observations and $z^{(\alpha)}$ is the number of observations that fell in the interval with proportion $\alpha$. Figure 8 depicts the reliability evaluation results for the predictive distributions obtained in the following 200 hours after the last training point and for the complete test set. The diagrams are for the complete next-day horizon (hours 19 to 42). This means that all probabilistic forecasts for all look-ahead times were used with equal weight when calculating the deviations. 


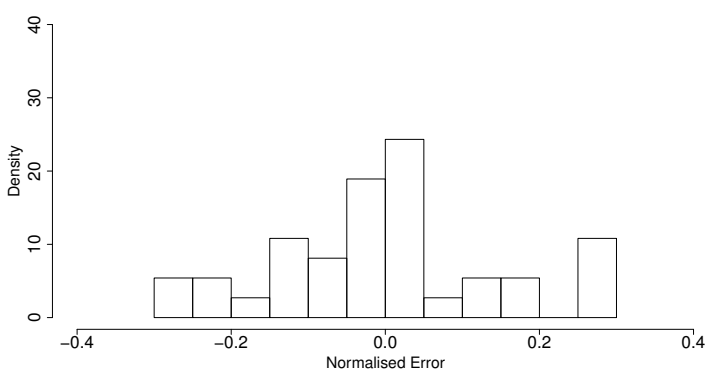

(a) Error at $t+19$

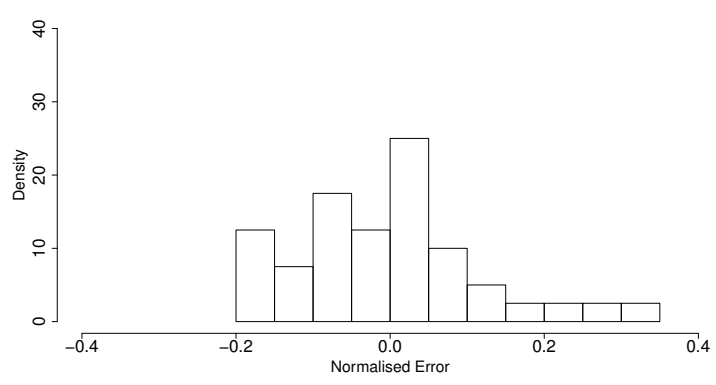

(b) Error at t +42

Fig. 6: Empirical distributions of the prediction error obtained with $v_{1}$ for two look-ahead times. Prediction errors are normalised on a scale $[-1,1]$.

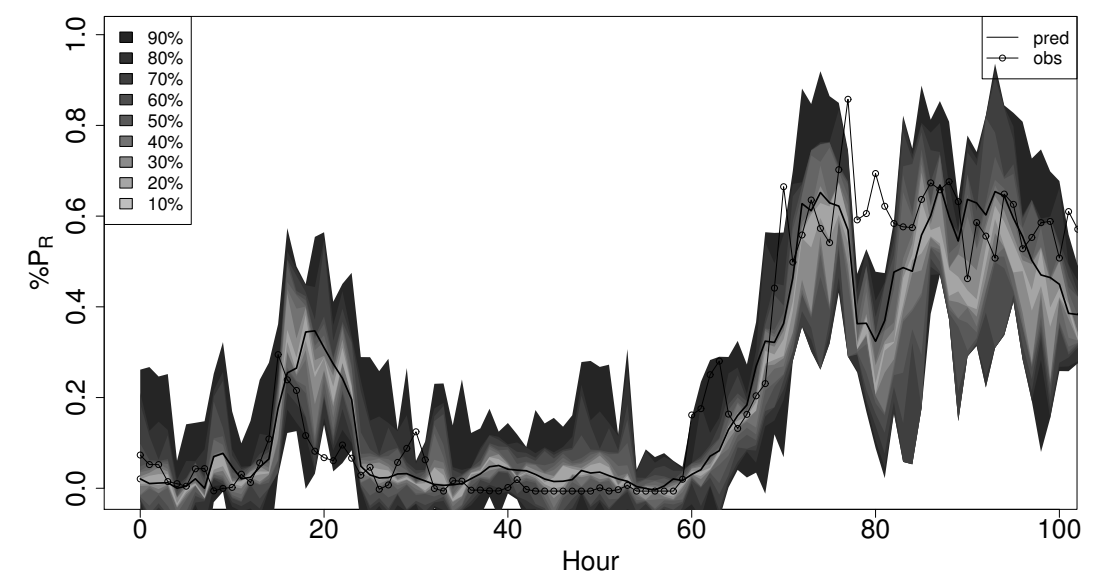

Fig. 7: Wind power point predictions and the associated interval forecasts using the closest point from the grid $\left(v_{1}\right)$

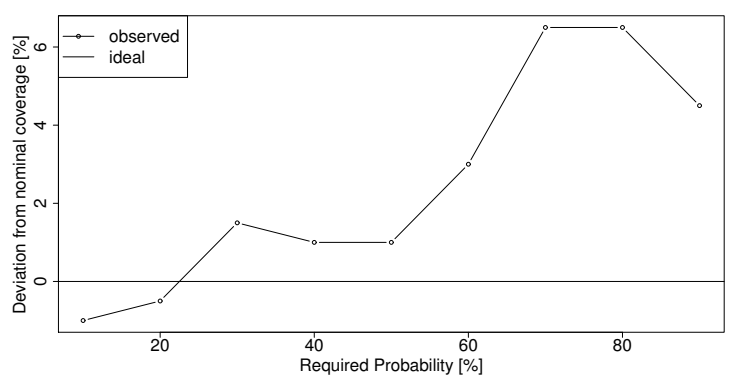

(a) Reliability on the first 200 hours of the test set

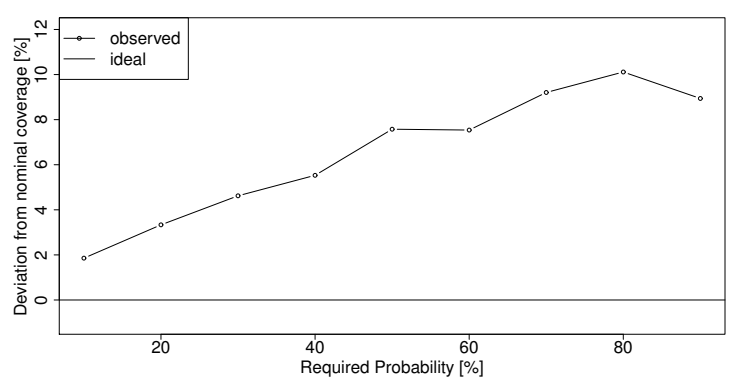

(b) Reliability over all the test set

Fig. 8: Reliability diagrams of 5, .., 95 percentiles estimations made with the Quantile Regression Forest procedure. 
From Figure 8 there are a couple of things to note. The narrower intervals (10\% and 20\%) tend to be overestimated on the first 200 hours, having a slightly higher coverage, while the larger intervals tend to be underestimated. In the same figure, diagram $b$ shows how the reliability decreases as the horizon increases. The results confirm the behaviour that was expected. The intervals are valid only for a short period of time after the training period. In order to maintain the reliability, the GP model would need to be retrained as new information becomes available and the error distribution needs to be recalculated. This would require further study.

Most existing prediction methods provide point forecasts and, more recently, a way to calculate their uncertainty. However, there are situations where a forecast from a single source does not provide enough information, specially during extreme situations where power system operators need to handle large deviations of power generation. There is a growing interest in the use of ensembles in order to improve point forecasts and to develop uncertainty models from these. However, it has been shown that NWP models are capable of modeling these events but with a misplacement error [26]. The following section presents two approaches for ramp characterisation based on the incorporation of the error distribution into the ramp detection algorithm and the use of a Fuzzy Inference System (FIS) to avoid the crisp classification.

\section{Power Ramp Characterisation}

One of the current challenges in wind power forecasting is the ability to handle extreme events, which can represent a different situation depending on the end user. In general, a ramp event is a rapid change in power output, either increase or decrease, within a small time window. Whether to use an increase of $50 \%$ or $30 \%$ of nominal power is, as mentioned before, up to the system operator. Wind power forecasts usually model very well the increases or decreases that happen within large time windows (at a small change rate). However, changes in small time windows are more difficult to model correctly. Cutler et al. [26] studied the weather phenomena that were causal of ramp events. They found that the majority of the events that were studied were associated with cold fronts, low pressure systems and troughs, which are well modeled by the numerical model but might be placed in the wrong physical position.
Another factor that might contribute to the inadequate characterisation of ramp events is the crisp definition of such events. In the literature, most studies are based on a binary definition, where the ramp is defined as a specific percentage of change in a specific time window. With this definition, events which might be slightly lower in change might not be identified but could be equally important in the eye of the operator. A good characterisation strategy should be more flexible and able to identify small but potential events.

The approach presented in this paper incorporates two different aspects. One is the incorporation of the uncertainty error information into the ramp detection algorithm and the use of fuzzy rules to classify the events. The other is the use of multiple grid points to address the misplacement, expecting that other gird points could model some events that were not well modeled using the closest point.

The basic ramp approach with no error consideration using fix percentage change and window size proceeds as follows:

1. The percentage $p$ of the amount of change in power output and the maximum window size $w$ are set.

2. The current sliding window size is set to 1 .

3. Using the current sliding window size, the power signal is analysed to identify any increase (rampup) or decrease (ramp-down) by $p$ percent. As soon as a ramp is detected, this is identified as the start time of the ramp and the end of the ramp will be identified in the following hours as soon as the ramp changes direction.

4. In the final set of ramps identified, if any two are overlapped, they are considered as one ramp, and the start and end times are readjusted.

5. The size of the window is increased one hour.

6. If the window size has not reached its maximum +1 , then go to step 3 , else, check any overlaps and output the identified ramps.

The algorithm was applied on both forecasted and real power output of the farm to identify the falsely and truely forecasted ramps. The time window was set to 5 hours, according to [24]. The percentage of change was set to $30 \%$ due to a very small number of real events of higher change. A total number of 21 ramps were observed in the available data from the month of April. Of the 21 ramps observed, 8 of them where forecasted accurately in direction (ramp up or down) and with a phase error less than \pm 12 hours. This time period 
of association is the maximum time difference between the timing of the forecast and the observed ramps, according to [24], that can maintain realistic connection between the forecast and the observed event. Events further apart might be representing totally different events. The total ramp accuracy and ramp capture percentage were calculated using equations 3 and 4 .

ramp capture $=\frac{\text { true forecasts }}{\text { true forecasts }+ \text { missed ramps }}$

forecast accuracy $=\frac{\text { true forecasts }}{\text { true forecasts }+ \text { false forecasts }}$

True forecasts are those forecasted ramps that are associated with an observed ramp; false forecasts are those ramps that were forecasted but did not occur; finally, missed ramps are those observed events that were not forecasted. Figure 9 shows an example of a true forecast of a ramp up that occurred on the 9th of April. Although the observations indicate that the ramp started around 10:00 hrs, the forecast was able to model this event one hour later, from 11:00 hrs to 02:00 hrs the next day. One can also notice that the fluctuation at $10 \mathrm{am}$ was not modeled. It might have been caused by local conditions that were not captured at the mesoscale level, however the increase tendency was modeled effectively.

Table 2 shows the results of the approach described previously (left) as well as results obtained with a second approach that takes into account the error (right). This approach will be introduced later on in the paper. Focusing on the results at the left, it can be observed that the capture percentage obtained from the forecast is quite low. However, the result is not reflecting what could be appreciated in the signal, as some of the ramps that were not detected by the algorithm were about $3 \%$ under the threshold. The ramps would have been detected using a smaller percentage of change that could be equally important to the operator. However, taking into account the current definition of a ramp implemented in the algorithm, only those ramps that are of exactly $30 \%$ or more can be detected. Other ramps that were not captured had an error between the 5 and $10 \%$, which can be attributed to the known underestimation of the forecast. If the estimation of the error was considered in the process, it could improve the ramp capture.

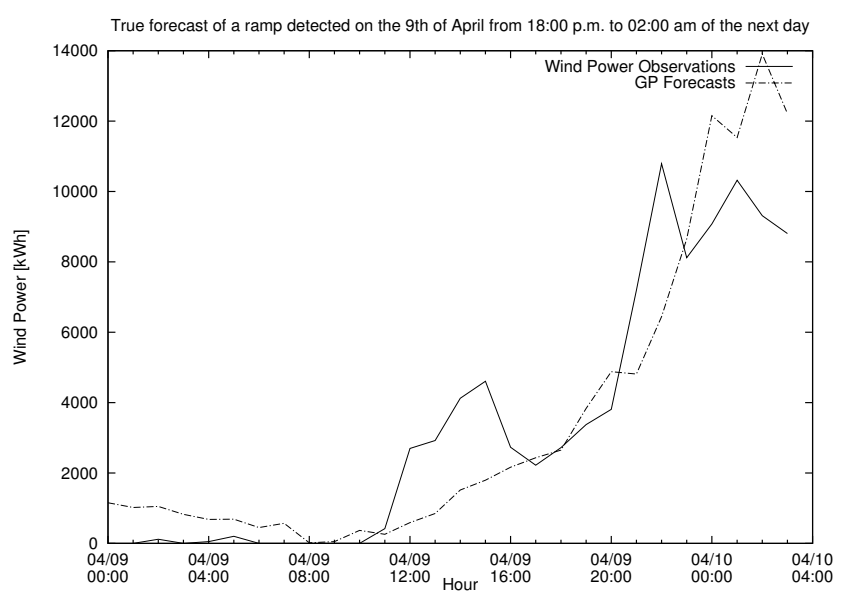

Fig. 9: True ramp forecasted on April 9th, 2012.

Table 2: Ramp frequency and forecast accuracy on April 2012 at Sotavento using the model at the closest point

\begin{tabular}{lll}
\hline & $\begin{array}{l}\text { Basic } \\
\text { approach }\end{array}$ & $\begin{array}{l}\text { Considering } \\
\text { the error }\end{array}$ \\
Number of true forecasts & 8 & 15 \\
Number of false forecasts & 3 & 5 \\
Number of missed ramps & 14 & 7 \\
Forecast Accuracy & $72.72 \%$ & $75 \%$ \\
Ramp Capture & $36.36 \%$ & $68.18 \%$ \\
\hline
\end{tabular}

\subsection{Incorporating the forecast error distribution}

According to the previous analysis, the error of the model on the training set is most of the time negative (over estimation). However, the histograms show some large under estimation errors. This could be potentially related to the fact that the GP algorithm works by minimising the root mean squared error (RMSE), which during ramp events where there are timing errors might result into high penalisations. For this reason, it could be expected that some large changes in power output would be missed in subsequent forecasts. To investigate the potential of using the error distribution for the ramp characterisation, the ramp detection algorithm presented previously was adapted to incorporate the error during the calculation of the change percentage on the sliding window. The possible errors on the start and end of the window were taken into account only if by incorporating the error, the difference between start and end point increased. It is important to make clear that so far the error distribution is not time dependent, each hour is treated independently. 
Results with this approach are shown also in Table 2. The ramp capture increases significantly and there is not much increase in the number of false forecasts, keeping the ramp accuracy at a sufficient level. These accuracy measurements are obtained using the binary definition on the real power output. This could mean that ramps that are apparently false forecasts could actually be "almost" a ramp on the real power output. This situation is shown on Figure 13. The figure shows a ramp up and ramp down which are not at the 30\% change on the observations. The figure also shows four different forecasts using four different points from the grid. Most of the models show an increase and decrease which are identified as a ramp events. Although in the real power output this was not identified as a ramp due to the binary definition, it could still be of impact, if it is just slightly lower.

In order to avoid these "false" captures, the binary definition of ramp events could be relaxed and changes could be categorised or scored according to certain criteria. To do this, a fuzzy rule based approach is proposed. This will allow to also consider those events, which are likely to become ramp events.

\subsection{A Fuzzy Logic approach for ramp characterisation}

Fuzzy set theory, proposed by L.A. Zadeh, provides a methodology that allows to deal with the imprecision of practical systems [40]. In a given system, where an output is produced according to certain inputs, those inputs or elements may have different states or values which represent ranges. This ranges which are not precisely defined, can be modeled using fuzzy sets. To decide whether the element belongs to one or another set, a membership function is used. The membership functions depict the degree of membership or one-to-one correspondence between an element in a domain and a truth value. Membership functions take the form

$\mu_{A}(x) \leftarrow f(x \in A)$

where $\mu_{A}$ is the membership function and $x$ is an element of the set $X$, which may belong to a fuzzy set $A$. The membership functions may have different shapes according to the experience of the designer.

Once the input is mapped to a set, the process of deciding what the output should be is done by using a set of rules. Fuzzy rules describe in a high level language how elements of the domain, which are inputs to the system, map to the outputs. These are a set of IFTHEN rules that are applied when an input has been mapped to its fuzzy set with the membership function. After the evaluation of these rules, a fuzzy set associated with each model solution variable is produced. Then, a process of defuzzification is used to find the value that best represents the information contained in the fuzzy set. This value is called the FIS score.

In terms of ramp characterisation, the decision to be made is whether a change in power is a potential ramp event. The binary classification can be avoided by characterising the event as a high, medium or low probable ramp event without discriminating it completely. The variables that are used in the decision making process, as well as the rules, are chosen by and depend on the knowledge and expertise of the designer. The experience of power grid operators would need to be taken into account in order to adjust the inputs, fuzzy sets and rules according to the situation of the grid. Here, the design decisions were based on the behaviour observed in past data. Two input variables are considered, the amount of change in power and the time window. There is an interesting relationship between these two variables that can provide information about ramp events. When the time window is small and a large change is observed, there is a high probability that the tendency could continue in the following hours, marking the start of a ramp event. Moreover, when the time window increases to a medium size $(2 \sim 4$ hours $)$, and if the change is high enough, there might still be a high possibility that a ramp event is happening. Finally, if the time window is at its maximum size and the change was close to the percentage of change that defines a ramp event (in this case 30\%), then it is definitely a ramp. On the other hand, there are situations which are less likely of developing a ramp event. Situations where the time window is high and the change is low, either the increase/decrease event is occurring very slowly or the event happened in a smaller window, so in both cases the probability is low. This is opposite to a low change in a small window which can potentially still be a ramp in the coming hours.

This behaviour was translated into fuzzy sets and rules. Figure 10 shows the two input variables and member functions used. The time window size uses a triangular member function, as it is the standard. The 

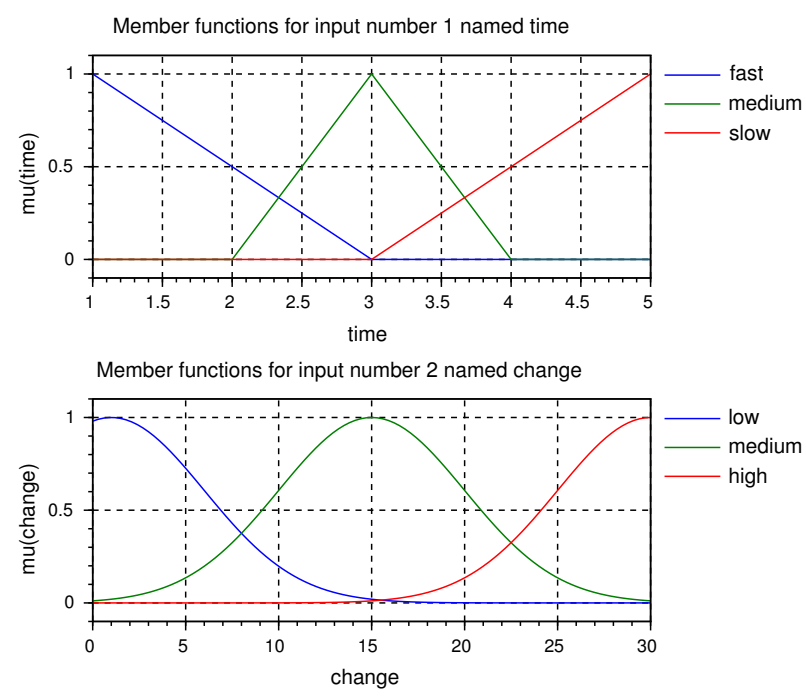

Fig. 10: Member functions for variables time and rate.

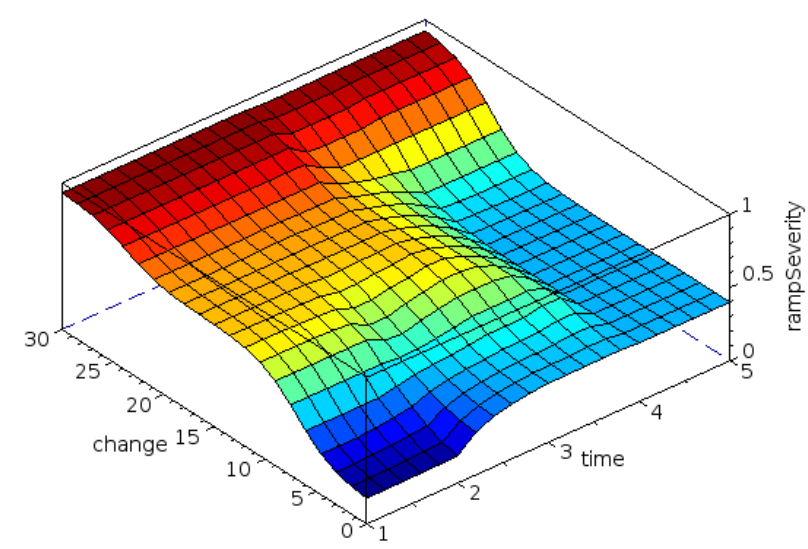

Fig. 11: Surface plot of the solution domain according to fuzzy rules.

power change rate implements a gaussian membership function, as this function has a softer transition between sets. It also naturally introduces a "shoulder" near zero, suppressing the influence of small changes without introducing a third free parameter. The rules are shown in Table 3. With these rules and the fuzzy sets, a more soft decision of what a ramp event is could be made, allowing the identification of smaller equally important events.

To test the fuzzy inference system, the forecast signal was filtered into 5 signals. The first signal is the result of calculating the differences in power output tak- ing a sliding window of one hour. The second signal was created taking the differences on sliding windows of two hours. The same process was repeated for the other window sizes. Each filtered signal is slightly shorter than the original due to the window size. Once the five filtered signals were obtained, each one was used individually as input to the fuzzy inference system. Each value of the filtered signal was taken as a new input, and the window size was chosen according to the filtered signal being tested. Results are shown in Figures 12. Colour maps were used to facilitate the interpretation of the results. As the human eye is sensible to the difference in colour, differences can be captured more easily than when results are presented as quantities. In addition, the shapes provide some interesting facts about the characteristics of the event. A maroon colour corresponds to a FIS score of 1, which represents a high severity. A light maroon/crimson colour corresponds to a mid-high severity which is between 0.7 and 0.9 score. The yellow/green corresponds to low severity events, while the blue colour is definitely not a ramp event. The ramp up and down events were separated into two graphs to avoid colour maps being too saturated. The top figures are the actual power signal, the figures in the middle show the ramp up events and the last row shows the ramp down events.

Focusing on the second line of the figure, the ramp up events, it can be observed that the largest change (between hour 60 and 80) is the strongest in FIS score along the 5 filtered signals. What the persistent colour in almost all filter signals means is that the change at the different time windows was very high and so was the total amount of change. The step shape of the line is due to the fact that at the very beginning of the event, the change on the following hour was slow but then suddenly increased in the following hours, marking a high score at the same start time but on the 5 th filtered signal. In addition, the band on the top ( 5 hour window) is wide, meaning that the change continued to increase even after the 5 hour window. If the event was shorter, strictly limited to 5 hours, the line would be thinner. This effect is not reflected for example on the change detected after hour 160. A straight line is shown in this case, meaning that from the very start of the event it increased with a very prominent slope. The ramp up observed at hour 80 is a false ramp due to large error on the forecast. The corresponding ramp down of this false ramp up can also be observed on 
Table 3: Rules

\begin{tabular}{lr}
\hline Rule 1. & If change is high then rampSeverity is high \\
Rule 2. & If time is fast and change is medium then rampSeverity is midhigh \\
Rule 3. & If time is medium and change is medium then rampSeverity is midhigh \\
Rule 4. & If time is slow and change is medium then rampSeverity is medium \\
Rule 5. & If time is fast and change is low then rampSeverity is medium \\
Rule 6. & If time is medium and change is low then rampSeverity is medium \\
Rule 7. & If time is slow and change is low then rampSeverity is low \\
\hline
\end{tabular}
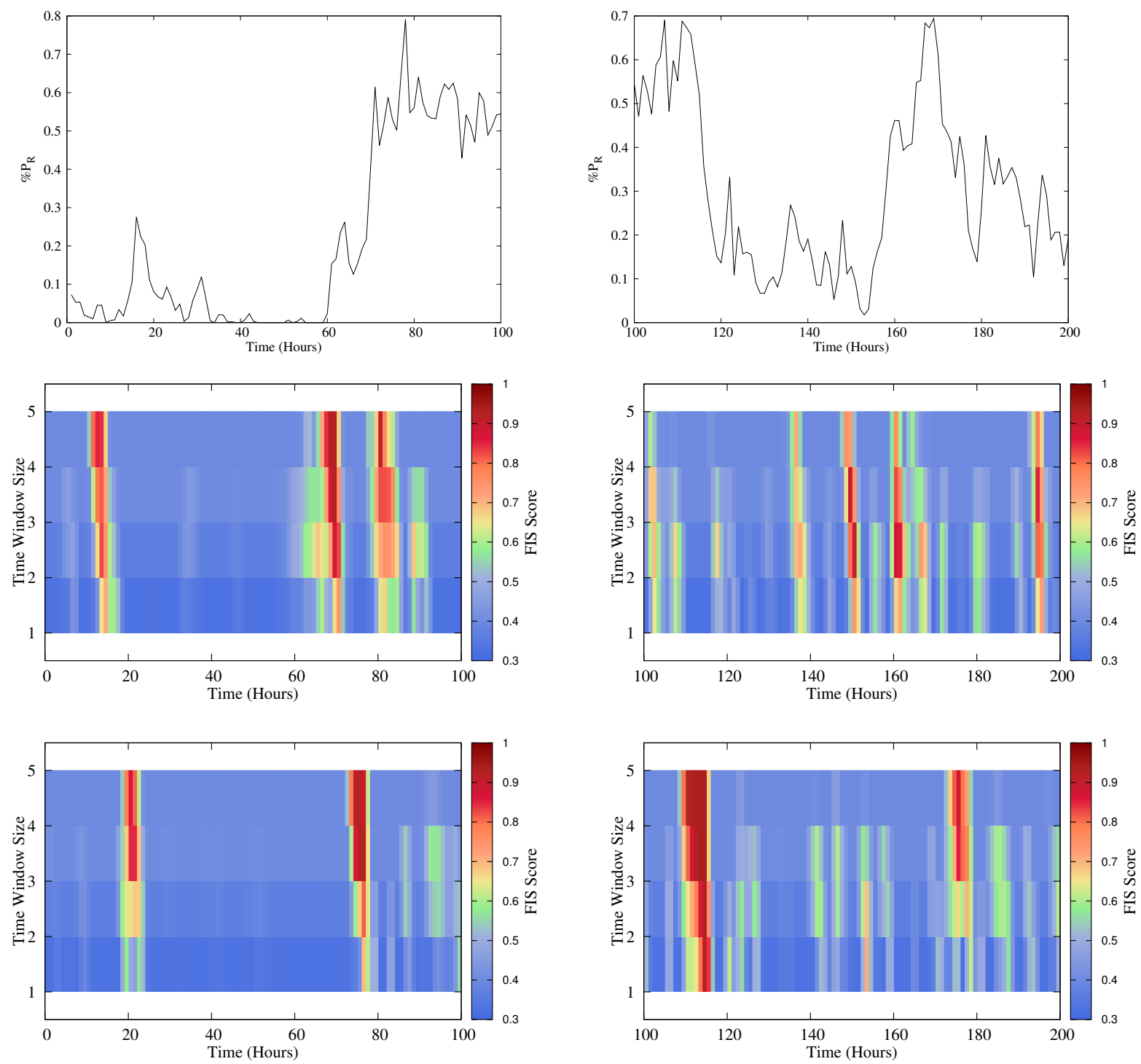

Fig. 12: Real power output and fuzzy inference system scores corresponding ramp up and ramp down events on the same time period. The graphs on the left correspond to the first 100 hours after the last training point. The graphs to the right correspond to hours 100 to 200 after the last training point. 
the bottom of the figure. Another interesting thing to mention is that the "false" ramp up before hour 20 is shown with a slightly lighter colour meaning that the total change in the 5 hour window did not reach the $30 \%$ change, nevertheless it is an important increase to take into account. Looking more into the bottom part of the figure, it can be observed that the largest ramp down events are correctly identified, at hours 120 and 180 . The original power signal has several small increases and decreases which can be seen in the filtered ones as noise.

All these characteristics that can be highlighted through the use of the colour maps can improve the identification of the ramps as more information about how the event will be developed is given. The results show in general the capacity of the fuzzy system to identify the events that were previously identified using the ramp detection algorithm, and in addition to this, presents some additional events which are categorised as potential ones. These additional events are presented in lighter colours as they represent less risk and therefore less probability of actually happening. It might still be possible that the forecast signal even with the error taken into account could not represent the real intensity of the event due to misplacement errors, and that other points of the grid could highlight better these changes. It would be interesting to see if exploring other closer locations could provide even more important evidence of these changes.

\section{Conclusions and Future Work}

In this paper, a novel approach to wind power ramp characterisation is presented. The approach introduces the use of the error distribution and fuzzy logic rules to improve the characterisation of ramp events which might not be identified by using a binary definition. According to the results shown in Table 2, the percentage of ramp capture improves as the error is taken into account and the introduction of fuzzy rules provides information about possible events which were not forecasted with a change of $30 \%$ but could potentially be of interest to the grid operator especially during periods of constant fluctuations.

Although the number of "false" ramps may increase with the fuzzy approach for ramp detection, it will be able to flag more potential events, which is equally important giving the operator the information to decide

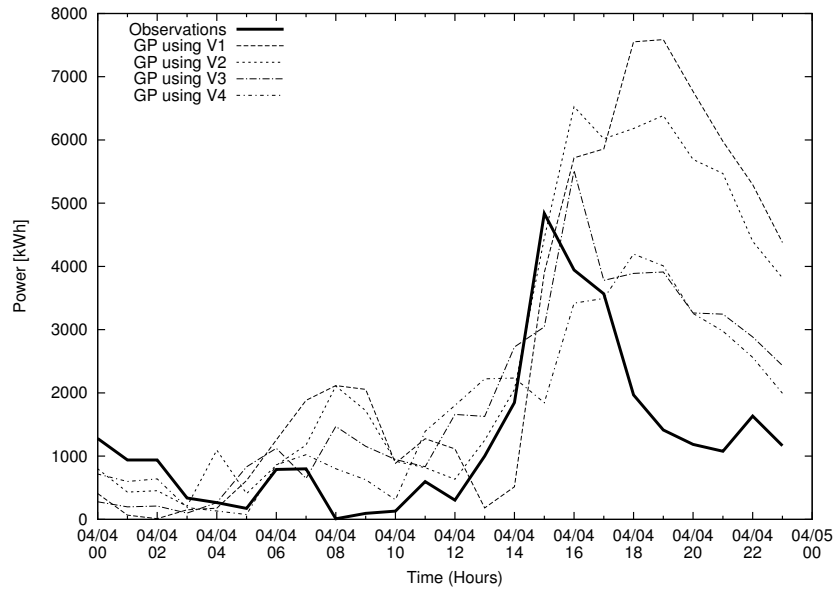

Fig. 13: Ramp on the 4th of April using the four grid points surrounding the farm.

whether it should be considered a ramp event or not. An interesting point to address in future work is the use of neighbour points to provide an estimation of the start and end time of the event. Preliminary results as the one in Figure 13 show how an event can be modeled differently considering different surrounding points. Figure 14 shows some preliminary results applying the fuzzy approach to these points. In the figure, the closest point (top right) and two neighbour points on the same period of time are presented. In general, there is a match in the three points about the events shown, although they present different intensities. The interesting aspect to see in this figure is the detection of two events around the 300th hour by the second neighbour (bottom right). These frequent changes in power output were not well modelled by the closest point of the grid. However, a neighbour point to the west of the wind farm is starting to represent these events which are not well located in time but they can indicate a period of various changes that might be important to considerate from the system operator's point of view. This is consistent with the wind direction observations which in general come from the southwest of the farm.

It is important to take into account that these results are related specifically to the Sotavento Experimental Wind Farm. Each wind farm has different terrain characteristics that affect the power production of the farm in different ways. The advantage of GP is that no assumptions about the model are needed. This does not mean that the model could not be improved with 

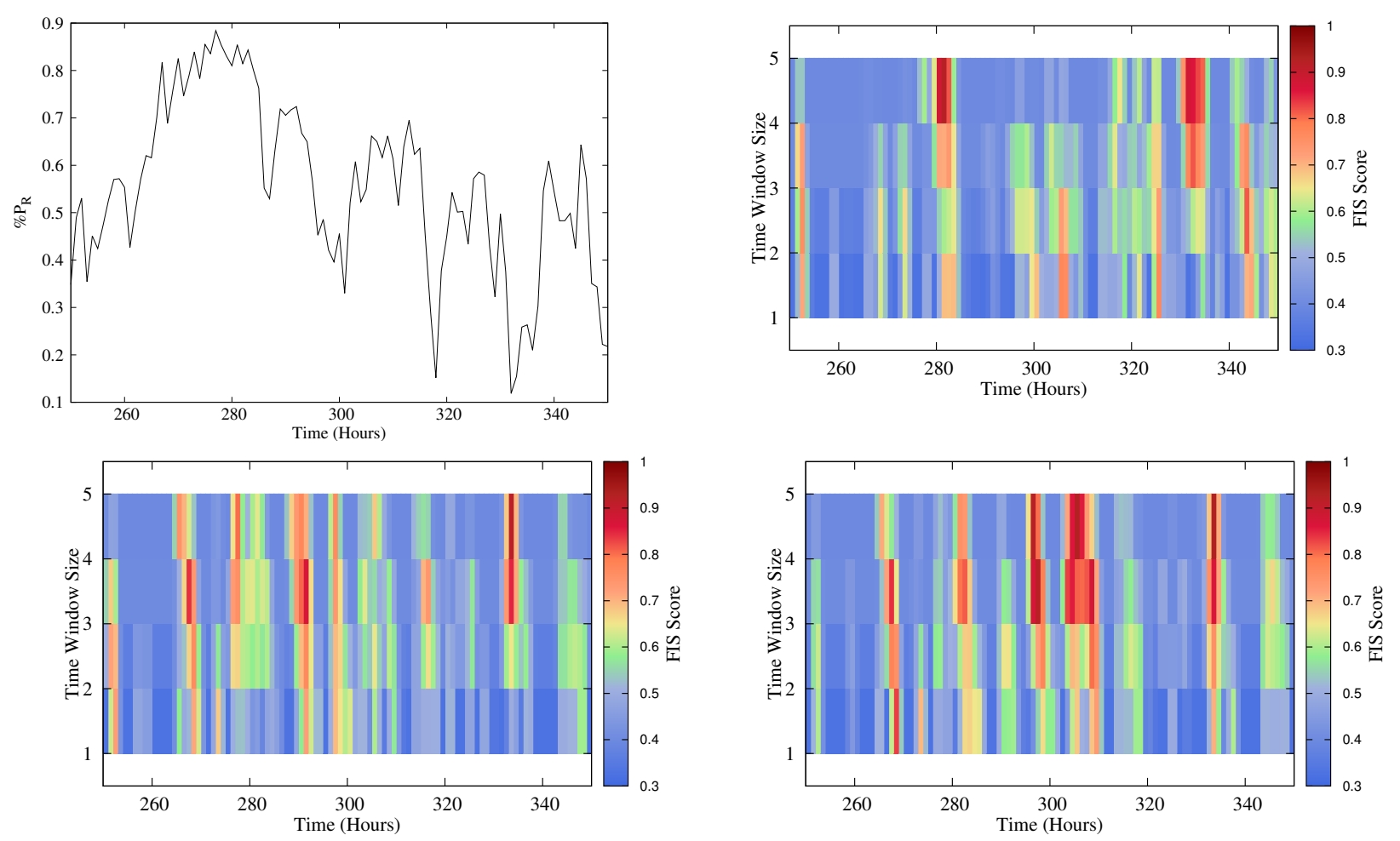

Fig. 14: Real power output and fuzzy inference system scores corresponding ramp down events at three different points on the same time period ( 250 to 350 hours after the last training point).

local information, specially for the smaller fluctuations. However, for a day-ahead forecast, this approach provides a wider picture of the possible events at the farm. Other variables, such as temperature and pressure, will be explored as a way to improve the fuzzy inference model during periods of smaller frequent fluctuations as these are usually associated with sudden changes in power output. Finally, from the system operator's point of view, the fuzzy logic approach would allow a better understanding of how the events will develop over time. Future work will also focus on providing an easier way to interpret the results and how to combine the fuzzy ramps from different points into one forecast, which would also be easier to interpret by the system operator.

\section{References}

1. WWEA, World wind energy half-year report 2012, Tech. rep. World Wind Energy Association (2012).
2. Ferreira, C., Gama, J., Matias, L., Botterud, A. and Wang, J. A Survey on Wind Power Ramp Forecasting, Tech. rep. ARL, DIS-10-13 (2010).

3. Pinson, P. Catalogue of complex extreme situations. Technical Report, EU Project SafeWind, Deliverable Dc1.2, (2009).

4. Giebel, G. The State of the Art in Short-Term Prediction of Wind Power: A Literature Overview, 2nd Edition, Project ANEMOS. Available at http://www.safewind.eu/images/Articles/Deliverables/ swind_deliverable_dp-1.4_sota_v1.1.pdf [Accessed on September 17, 2013].

5. Kanamitsu, M. and Alpert, J.C. and Campana, K. A. and Caplan, P.M. and Deaven, D.G. and Iredell, M. and Katz, B. and Pan, H. L. and Sela, J. and White, G. H., Recent Changes Implemented into the Global Forecast System at NMC, Weather and Forecasting, Vol.6, 1991, pp. 425-435.

6. Landberg, L., Short-term prediction of the power production from wind farms, Journal of Wind Engineering and Industrial Aerodynamics, Vol. 80, 1999, pp. 207-220.

7. Landberg, L., Short-term prediction of local wind conditions, Journal of Wind Engineering and Industrial Aerodynamics, vol. 89, 2001, pp. 235-245.

8. Constantinescu, E.M., Zavala, E.M., Rocklin, M., Sangmin Lee, Anitescu, M., A computational framework for un- 
certainty quantification and stochastic optimization in unit commitment with wind power generation, IEEE Transactions on Power Systems, vol. 26, 2011, pp. 431- 441.

9. Skamarock, W.C. and Klemp, J.B. and Dudhia, J. and O. Gill, D. and Barker, D. M. and Wang, W. and Powers, J. G., A Description of the Advanced Research WRF Version 2, AVAILABLE FROM NCAR, Vol.88, 2001, pp. 7-25.

10. Alexiadis, MC., Dokopoulos, PS., Sahsamanoglou, H., Manousaridis, IM., Short-term forecasting of wind speed and related electrical power, Solar Energy, Vol. 63(1),1998, pp. 61-68.

11. Lazić, L. and Pejanović, G. and Živković, M., Wind forecasts for wind power generation using Eta model, Renewable Energy, Vol.35, No.6, 2010, pp.1236-1243.

12. C. Monteiro, R. Bessa, V. Miranda, A. Botterud, J. Wang, G. Conzelmann, I. Porto, et al., Wind power forecasting: state-of-the-art 2009., Tech. rep., Argonne National Laboratory (ANL) (2009).

13. Costa, A., Crespo, A., Navarro, J., Lizcano, G., Madsen, H. and E. Feitosa. A review on the young history of the wind power short-term prediction. Renewable and Sustainable Energy Reviews, vol 12, no. 6, pp. 1725 - 1744 (2008).

14. A. M. Foley, P. G. Leahy, A. Marvuglia, E. J. McKeogh, Current methods and advances in forecasting of wind power generation, Renewable Energy, vol. 37, no. 1, pp. 1 8 (2012). http://dx.doi.org/10.1016/j.renene.2011.05.033.

15. Sweeney, C.P. and Lynch, P., Adaptative post-processing of short-term wind forecasts for energy applications, Wind Energy, doi. 10.1002/we.420 (2010).

16. Zhao, P. and Wang, J. and Xia, J. and Dai, Y. and Sheng, Y. and Yue, J. Performance evaluation and accuracy enhancement of a day-ahead wind power forecasting system in China, Renewable Energy, Vol.43, pp. 234-241(2012).

17. Delle Monache, L. and Nipen, T. and Liu, Y. and Roux, G. and Stull, R. Kalman Filter and Analog Schemes to Postprocess Numerical Weather Predictions, Monthly Weather Review, Vol.139, No.11, pp. 3554-3570 (2011).

18. Cassola, F. and Burlando, M., Wind speed and wind energy forecast through Kalman filtering of Numerical Weather Prediction model output, Applied Energy, Vol.99, pp. 154-166 (2012).

19. Salcedo-Sanz, S. and Ortiz-García, E. G. and PortillaFigueras, A. and Prieto, L. and Paredes, D, Hybridizing the fifth generation mesoscale model with artificial neural networks for short-term wind speed prediction. Renewable Energy, vol.34, no.6, pp.1451-1457 (2009).

20. Salcedo-Sanz, S. and Ortiz-García, E. G. and PérezBellido, A. M. and Portilla-Figueras, A. and Prieto, L., Short term wind speed prediction based on evolutionary support vector regression algorithms, Expert Systems with Applications, vol.38, no.4, pp. 4052-4057 (2011).

21. Bourke, W. Performance of the ECMWF and the BoM Ensemble Systems in the Southern Hemisphere. Monthly Weather Review, vol. 132, pp. 2338-2357 (2004).

22. Toth, Z. and Kalnay, E. Ensemble Forecasting at NMC: The Generation of Perturbation. Bulletin of the American Meteorological Society, vol. 74, pp. 2317-2330 (1993)..

23. Arribas, A., Robertson, K. B. and Mylne, K. R. Test of a Poor Man's Ensemble Prediction System for Short-Range Probability Forecasting. Monthly Weather Review, vol. 133, pp. $1825-1839$.
24. Greaves, B., Collins, J., Parkes, J., Tindal, A. Temporal forecast uncertainty for ramp events. Wind Engineering, vol. 33, no. 11, pp. 309-319 (2009).

25. Bossavy, A., Girard, R. and Kariniotakis, G., Forecasting ramps of wind power production with numerical weather prediction ensembles, Wind Energy, vol. 16, no. 1, pp. 5163 (2013)

26. Cutler, N.J., Outhred, H.R., MacGill, I.F., Kepert, J.D., Characterizing future large, rapid changes in aggregated wind power using numerical weather prediction spatial fields. Wind Energy, vol. 12, no. 6, pp. 542-555 (2009).

27. Gallego, C., Costa, A., Cuerva, A., Landberg, L., Greaves, B and Collins, J. A wavelet-based approach for large wind power ramp characteristaion. Wind Energy, vol. 16, no. 2, pp. 257-278 (2013).

28. Martínez-Arellano, G. and Nolle, L. Genetic Programming for Wind Power Forecasting and Ramp Detection. Research and Development in Intelligent Systems XXX, pp. 403 - 417 (2013).

29. Koza, J.R., Genetic Programming: on the programming of computers by means of natural selection, MIT Press (1992).

30. Poli, R. and Langdon, B. and McPhee, N. F., A field guide to genetic programming with contributions by J. R. Koza, Published via http://lulu.com and freely available at http://www.gp-field-guide.org.uk, (2008).

31. Kotanchek, M. E. and Vladislavleva, E. Y. and Smits, G. F., Genetic Programming Theory and Practice VII, Springer US ( 2010).

32. Skamarock, W.C. and Klemp, J.B. and Dudhia, J. and O. Gill, D. and Barker, D. M. and Wang, W. and Powers, J. G., A Description of the Advanced Research WRF Version 2, AVAILABLE FROM NCAR, Vol.88, pp. 7-25 (2001).

33. J. Michalakes, M. Vachharajani, GPU Acceleration of Numerical Weather Prediction, Parallel Processing Letters, Vol. 18 No. 4 . World Scientific. Dec. (2008) 531 - 548.

34. Martínez-Arellano, G., Nolle, L. and Bland, J. Improving WRF-ARW Wind Speed Predictions using Genetic Programming. SGAI'12 Conf. pp 347-360 (2012).

35. Tindal, A., Johnson, C., LeBlanc, M., Harman, K., Rareshide, E. and Graves, A. Site-especific adjustments to wind turbine power curves. AWEA Wind Power Conf. (2008).

36. Sotavento Galicia Experimental Wind Farm, sotaventogalicia.com, accessed on 29 April, 2013.

37. Pinson, P. Estimation of the uncertainty in wind power forecasting, PhD Thesis, Ecole des Mines de Paris, Paris, France (2006).

38. G.Kariniotakis and co authors. What performance can be expected by short-term wind power prediction models depending on site characteristics? In CD-Proc. of the 2004 European Wind Energy Conference, EWEC04, London, United Kingdom, November 2004

39. Meinshausen, N. Quantile Regression Forests. Journal of Machine Learning Research. Vol. 7, pp. 983 - 999 (2006).

40. L. A. Zadeh, Fuzzy Sets, Information and Control, New York: Academic Press, vol. 8, pp. 338-353 (1965)

41. Zhang, Y., Wang, J. and Wang, X. Review on probabilistic forecasting of wind power generation. Renewable and Sustainable Energy Reviews, vol. 32, pp. 255 - 270 (2014). 